\title{
Architecture et urbanisme postmodernes : une expression du relativisme contemporain?
}

Liliane Voyé

\section{OpenEdition}

1 Journals

Édition électronique

URL : http://journals.openedition.org/ress/542

DOI : $10.4000 /$ ress.542

ISSN : 1663-4446

Éditeur

Librairie Droz

\section{Édition imprimée}

Date de publication : 1 septembre 2003

Pagination : 117-124

ISBN : 2-600-00878-0

ISSN : 0048-8046

\section{Référence électronique}

Liliane Voyé, «Architecture et urbanisme postmodernes : une expression du relativisme contemporain?", Revue européenne des sciences sociales [En ligne], XLI-126 | 2003, mis en ligne le 30 novembre 2009, consulté le 10 décembre 2020. URL : http://journals.openedition.org/ress/542 ; DOI https://doi.org/10.4000/ress.542 
Liliane VOYÉ

\title{
ARCHITECTURE ET URBANISME POSTMODERNES : UNE EXPRESSION DU RELATIVISME CONTEMPORAIN?
}

\begin{abstract}
Quelles que soient les réactions qu'inspire le terme de post-moderne, force est de constater qu'une tendance majeure de l'architecture et de l'urbanisme de la fin du $\mathrm{XX}^{\mathrm{e}}$ siècle et du début du $\mathrm{XXI}^{\mathrm{e}}$ siècle s'écarte de façon manifeste du courant moderniste qui a profondément marqué la période directement précédente et qui était venu rompre de façon volontairement radicale avec les traditions antérieures. Dans le champ concerné, le terme ne suscite pas le rejet qu'il rencontre souvent dans celui des sciences humaines. Apparu dès la fin du XIX siècle dans le domaine de la peinture, repris plus tard par la critique littéraire et ce particulièrement dans le monde anglo-saxon - le terme post-moderne se retrouve dès 1945 en architecture. C'est Hudnut, le président de l'école d'architecture de Harvard, qui avait accueilli Gropius dans cette université, qui l'utilise pour traduire sa déception face à ce qu'il stigmatise comme étant l'impuissance de l'architecture moderne à répondre de façon satisfaisante aux problèmes posés par la réorganisation des villes et des logements (Hudnut, 1945). Mais c'est en 1977 que le terme va marquer définitivement ce champ, avec l'ouvrage de Charles Jencks «Le Langage de l'Architecture Post-Moderne » (1979), où l'auteur affirme que l'architecture moderne faite de boîtes en verre et en acier est symboliquement morte le 15 juillet 1972 à St Louis, Missouri, lorsqu'il fut procédé à l'implosion de l'ensemble moderne de logements sociaux très décriés de Pruitt-Igoe. Critiquant le caractère dogmatique sinon totalitaire de l'architecture moderne, Jencks y oppose une architecture qui, sans d'ailleurs exclure le modernisme, est ouverte au pluralisme des styles, des formes et des références, en relation avec la diversité des cultures. Il constate d'ailleurs que, contrairement au modernisme qui s'est constitué en école et a élaboré sa charte (La Charte d'Athènes), le post-modernisme n'a pas d'école mais s'exprime à travers une série (a cluster) de tendances diverses telles que le vernaculaire, le métaphorique, l'historiciste, [...]. Ces propos de Jencks sont par la suite repris par l'architecte Eisenman qui considère que l'architecture moderne est associée à une image du monde où c'est l'artefact qui domine tout comme c'est le cas, estime-t-il, pour la «peinture du non-objet» comme celle de Mondrian ou pour la musique atonale de Schönberg ou de Webern; l'architecture moderne va dans le même sens non pas, dit-il, parce qu'elle est fonctionnelle mais parce qu'elle est auto-référentielle. Et Eisenman oppose celle-ci à l'architecture postmoderne qui, dit-il, «manifeste la perte de confiance dans la rationalité de l'homme en jouant sur la décomposition, la fragmentation, la citation et des principes d'ordonnancement non linéaires et non hiérarchiques » (Eisenman, 1976).
\end{abstract}


C'est sur ce fond polémique que, après avoir brièvement rappelé les caractéristiques majeures de l'architecture et de l'urbanisme post-modernes, en les comparant brièvement à celles du modernisme, je voudrais proposer ici quelques réflexions sur la question de savoir dans quelle mesure l'architecture et l'urbanisme post-modernes peuvent être vus comme exprimant le relativisme qui marquerait aujourd'hui nos sociétés. Si tel est le cas, comment interpréter celui-ci et quel sens politico-idéologique revêt-il?

\section{DES TRAITS MAJEURS DU POST-MODERNISME}

L'architecture moderne nous est familière: elle se rencontre dans toutes les villes et cela dans le monde entier - ce qui la fait d'ailleurs qualifier de «style international». Constituée de grands immeubles d'acier, de béton et de verre, elle offre des formes identiques quels que soient leurs usages: logements, bureaux, services, entreprises, voire même prisons occupent des tours et des barres indifférenciées dont Choay stigmatise «la réduction sémantique»(Choay, 1967), les formes s'autonomisant du sens et perdant leur statut de symboles pour devenir signes abstraits qui ne peuvent se comprendre qu'à travers l'adjonction d'un lexique lui aussi abstrait qui vient rompre lui-même avec le caractère concret des désignations antérieures: les «grands ensembles» se substituent aux maisons ou aux logements, les «espaces verts» aux parcs et jardins et les «axes de circulation» aux rues et aux boulevards (Choay, 1967). Cette architecture et cet urbanisme modernes sont, avant tout, pensés en vue de doter l'économique d'espaces opératoires, fonctionnellement spécialisés et conçus sur une mode bureaucratique, au sens webérien du terme. C'est en effet ce type de rationalité qui y domine: il en explique la préférence pour la syntaxe orthogonale, l'angle droit et la ligne droite, pour le matériau brut et nu que ne viennent cacher aucune décoration ni aucune couleur ajoutée si ce n'est éventuellement le blanc. Cette «pureté » et cette «vérité », comme il est dit, des formes, des lignes et des matériaux sont explicitement requises par les promoteurs de cette architecture - de Loos, qui ne voit dans la couleur que «barbouillages de criminels et de dégénérés », à Le Corbusier, pour qui «le goût de l'ornement renvoie au sauvage endimanché qui nous habite encore ». L'important usage du verre doit, quant à lui, répondre à un souci de clarté, qu'il s'agit d'entendre non seulement dans sa dimension physique concrète mais aussi comme moyen d'exprimer et de favoriser la transparence des activités, voir le fonctionnement démocratique de celles-ci.

La conception intérieure de ces immeubles se veut, elle aussi, pleinement rationnelle: chaque fonction a son espace propre et réciproquement et tout ce qui apparaît comme non directement et pragmatiquement utile est exclu. Ainsi pas de caves ni de greniers, décrits par Le Corbusier comme des lieux où l'on accumule des choses que l'on jettera demain - celles échappant à ce sort devant trouver une place ad hoc spécifique, de l'armoire à valises à l'espace de rangement pour les équipements d'hiver en été et d'été en hiver. Tous les espaces intérieurs se voient ainsi désigner une fonction particulière dont il est souhaité qu'elle s'exerce en référence aux règles de l'ergonomie, comme en témoigne le «modulor». 
A la fonctionnalité interne des bâtiments renvoie en outre celle de leur agencement: des espaces distincts sont réservés pour les quatre activités considérées comme résumant toute l'existence humaine - habiter, travailler, se récréer et circuler - ce qui conduit à la création d'espaces monofonctionnels, séparés les uns des autres comme des îles éparses et reliés par des axes de circulation, eux-mêmes hiérarchisés selon les types d'usages et d'usagers. La composition se veut claire et non ambiguë; prohibant souvent tout aménagement ultérieur et toute appropriation particulière, elle est conçue pour rester telle qu'elle et pour ne subir aucune modification - la rationalité qui la commande étant conçue comme certaine et définitive.

C'est une toute autre figure que présentent l'architecture et l'urbanisme postmodernes apparus plus ou moins dans le dernier quart du vingtième siècle, même s'il s'en trouve diverses illustrations bien antérieurement. La dé-différenciation est la caractéristique majeure du post-modernisme et, avec elle, l'éclectisme, la conciliation de tous les styles, de toutes les références, de toutes les époques et de toutes les spécificités locales. Le post-modernisme redécouvre ainsi nombre d'archétypes architecturaux : la colonne et le fronton, le toit à pans coupés et les murs décalés, [...]. Il emprunte tout autant à l'architecture de Palladio qu'à celle du «strip» de Las Vegas et ne fait pas de distinction entre compositions savantes et formes populaires, références sacrées et inspiration commerciale. Et c'est bien souvent sur le mode de la citation qu'il use de ces différentes sources dont il emprunte des fragments pour les composer dans d'autres contextes. Loin que ne soit adopté l'impératif de vérité des matériaux, de pureté et de rationalité des formes qui commandait le modernisme, la couleur et l'ornement sont ici valorisés en ce qu'ils suscitent l'émotion et en appellent aux sentiments. La spécialisation fonctionnelle des espaces est abandonnée au profit d'une mixité et d'une complexité flexibles et mouvantes: espaces intérieurs ouverts et polyfonctionnels et «recomposables » indéfiniment; non-séparation des fonctions mais au contraire imbrication et entremêlement, notamment des lieux de résidence, de travail et de loisirs. Ainsi en témoigne, par exemple, la récente construction d'une usine d'automobiles Volkswagen en plein centre de la vieille ville de Dresde en Allemagne; en circulant pour faire leurs courses ou se rendre à l'école ou au travail les habitants voient les chaînes de montage et ils peuvent utiliser le piano-bar et les salles de conférences, y assister à des concerts et y visiter des expositions. Dans une même valorisation de la mixité, les nouvelles gares, en particulier celles conçues pour des arrêts TGV, se veulent multifonctionnelles, comme en témoigne par exemple la gare de Lille-Europe, où la fonction spécifique de gare se combine avec un centre d'affaires et de services, un centre commercial, des équipements de culture et de loisirs - tout cela étant conçu en interaction avec le centre urbain historique tout proche, l'ensemble étant destiné à devenir un vaste espace ludique et touristique (Bourdin, 2002: 12).

Ce souci de mixité conduit en outre l'urbanisme post-moderne à redécouvrir la rue avec la continuité de bâtir qu'elle implique. Avec aussi sa traditionnelle polyfonctionnalité: loin de n'être qu'axe de circulation, souvent en outre spécialisé, elle se veut non seulement ouverte à la diversité des trafics - piétons, vélos, voitures - mais aussi lieu de vie et de rencontre, lieu où les gens se frottent les uns aux autres, où le quotidien se donne en spectacle et où peut à chaque instant surgir 
l'événement. Loin qu'il ne soit vide ou espace-déchet, le tissu intercalaire prend quant à lui un sens plénier: il ne s'agit pas de relier plus ou moins artificiellement des fonctions indépendantes l'une de l'autre mais bien de les articuler et de doter de sens cette articulation et les accès progressifs de l'une à l'autre par des aménagements permettant soit d'accueillir des activités, soit de discourir sur des pratiques de l'espace. L'exemple de nombreux aménagements de giratoires aux entrées des villes et villages témoigne clairement de cette volonté: il s'agit d'en faire des «portes », avec toute la symbolique que Simmel attribue à celles-ci: elles relient le dedans et le dehors, opèrent des distinctions dans «l'uniformité continue » et expriment «la possibilité de briser à tout instant les limitations pour gagner la liberté » (Simmel, 1988: 163-166).

L'architecture et l'urbanisme post-modernes proposent aussi un rapport spécifique au temps et à l'espace. Là où le modernisme entendait faire table rase des formes du passé, jugées inadaptées et désormais non signifiantes, et y substituer des formes radicalement nouvelles conçues pour l'avenir, le post-modernisme n'hésite pas, bien au contraire, non seulement à garder et à préserver des formes anciennes mais aussi à se les approprier et à opérer des transferts d'usages. Ainsi Bofill a-t-il construit, à Saint-Quentin-en-Yvelines, un bâtiment sur le principe de l'aqueduc et, à Marne-la-Vallée, un immeuble qui prend la figure d'un amphithéâtre. Mais ces formes empruntées au passé cachent des fonctions tout autres que celles auxquelles elles renvoyaient à l'origine puisqu'elles abritent l'une et l'autre des logements sociaux. La réduction sémantique que Choay attribuait au modernisme prend ici une autre tournure puisque la forme ne renvoie plus à la fonction à laquelle elle était destinée; la symbolique se brouille tout comme le temps perd toute épaisseur dans un jeu parodique dominé par l'image.

De même qu'il s'amuse avec le temps, le post-modernisme triche en quelque sorte avec l'espace. Tournant le dos à l'uniformité universelle de l'architecture moderne, il redécouvre la diversité des formes, des matériaux et des compositions et leur association à des lieux particuliers. Mais à l'enracinement qui les caractérisait et qui contribuait à la construction et à la reconnaissance d'identités chaque fois particulières, fait place la délocalisation: partout peuvent se trouver des fragments empruntés à des lieux divers, assemblés en des endroits qui leur sont étrangers et où, à première vue, rien - ni les ressources matérielles locales, ni le climat, ni le mode de vie - n'en explique l'origine. Cette réaffirmation éclectique des lieux conjuguée avec leur délocalisation semble venir ainsi tout à la fois souligner la diversité et la spécificité des lieux et des cultures mais en les rendant accessibles et reconnaissables partout. L'architecture et l'urbanisme post-modernes paraissent ainsi à leur façon témoigner de la globalisation du monde. Comme les hommes, les cultures sont mobiles et non plus coincées sur le territoire qui leur était propre. Elles se répandent et se diffusent sur un plan universel mais souvent elles ne sortent pas indemnes de ces déplacements car le sens originel qui les soustendait est vécu dans un rapport d'extériorité qui le transforme, opacifie leur logique d'ensemble et risque parfois de réduire leur essence symbolique à une imagerie ludique. 


\section{POST-MODERNISME ET RELATIVISME}

Partout et depuis toujours, l'architecture et l'urbanisme expriment les caractéristiques de la vie sociale; ils en disent les priorités et les pouvoirs, les contradictions et les valeurs. Leurs versions moderne et post-moderne n'échappent pas à cette constante: chacune d'elles renvoie à un moment particulier de l'histoire de la société occidentale, dont elle visualise le projet et l'image qu'elle construit d'elle-même. Avant d'élaborer quelque peu la question de savoir si le post-modernisme exprime le relativisme qui, pour d'aucuns, marque nos sociétés, il n'est pas inutile de dire quelques mots du sens qui sous-tend le modernisme.

Quoique ce courant se soit amorcé bien antérieurement, l'architecture et l'urbanisme modernes se sont particulièrement développés et diffusés à travers le monde durant les «Trente Glorieuses ». Tout comme aux orientations dominantes de l'économie capitaliste de l'époque, répondait l'idéal-type de «l' homo economicus», purement rationnel et radicalement dégagé de toute équation personnelle et émotionnelle, ces formes et compositions modernes, elles aussi à prétention rationnelle, renvoyaient au nouvel homme urbain, «l'Orgman», l'homme de l'organisation (Rosenberg, 1965). Ces systèmes de formes que Choay considère comme symboliquement appauvris, répondaient ainsi, pour Jencks (1977: 442-445), aux exigences d'un système politique - l'Etat national fort - et économique - l'entreprise fordiste - réduisant l'individu à des rôles passifs purement fonctionnels, commandés et même conditionnés par les énormes appareils bureaucratiques mis en place par ces deux instances. Cette architecture et cet urbanisme, qui entendaient faire table rase du passé, s'inscrivent pleinement dans l'opulence - certes relative et très inégalement distribuée - et dans l'optimisme d'une époque confiante en elle-même et en son devenir: tout ne pouvait qu'aller vers un mieux, grâce à la maîtrise croissante de l'homme sur l'univers et aux avancées de sa rationalité, épaulée par le développement de ses connaissances.

Dès la fin des années septante, cette vision confiante allait peu à peu vaciller, ébranlée par les effets des rigidités du capitalisme, entraînant récession, montée du chômage et réduction de la capacité des Etats d'assumer pleinement le rôle de providence qu'ils s'étaient dévolu. Ces bouleversements ne pouvaient que se répercuter sur la vision du monde, laquelle allait bientôt se reconstruire autour de l'idée de «société du risque» (Beck), marquée par l'incertitude et par la multiplicité de changements susceptibles de surgir partout, à tout moment et en tout domaine sur le mode météorologique, c'est-à-dire difficilement prévisibles à moyen sinon à court terme.

L'architecture et l'urbanisme ne sont pas restés indifférents à cette transformation; souvent, ils en ont même produit des signes avant-coureurs et ils en offrent une matérialisation évidente. L'éclectisme des styles, les emprunts disparates au passé, le déplacement des spécificités locales, les mixités fonctionnelles, [...] tout cela semble traduire l'abandon de l'exigence rationnelle au bénéfice des égarements d'un émotionnel peu contrôlé, trouvant à se déployer dans des formes et un environnement ludique, où tout est proposé comme équivalent.

Peut-on pour autant en conclure au relativisme de cette architecture et de cet urbanisme, pour lesquels tout serait bon et que n'organiserait aucun principe majeur? La réponse est moins évidente qu'il n'y paraît et, sans prétendre la 
fournir une fois pour toutes, je voudrais proposer quelques éléments qui peuvent être susceptibles de l'éclairer.

Tout d'abord, il paraît clair que le post-modernisme renvoie à une réalité devenue problématique ou, plus exactement, désormais perçue comme telle. Après un temps de certitude qui, dans une cécité dangereuse, jouait souvent avec l'utopie, on est en effet entré dans un monde où, comme le dit Comte-Sponville, se pose « la question de savoir qui maîtrise la maîtrise [...] parce que la science et la technique ne donnent aucun moyen de maîtriser ni la science, ni la technique [...]. C'est le problème des valeurs » (Comte-Sponville, 1999: 34-35). Le messianisme scientifique qui a marqué le XIX $\mathrm{X}^{\mathrm{e}}$ siècle et les trois premiers quarts du $\mathrm{XX}^{\mathrm{e}}$ siècle a fait place au doute et à une rationalité nouvelle qui tient désormais compte de la liberté de choix et ouvre ainsi sur divers possibles (Prigogine, 1998: 75-76). Ce qui, à première vue, apparaît ainsi comme du relativisme traduirait en fait l'affirmation de la liberté de l'homme, avec ce qu'elle porte en elle d'anxiété (Sartre ne disait-il pas qu'il est plus facile d'être esclave que maître...) et d'exigence constante de choix avec ce que celui-ci suppose toujours de risque. L'éclectisme de l'architecture postmoderne pourrait ainsi vouloir dire qu'aucune forme ne s'impose comme telle de façon absolue parce que les représentations des hommes sont multiples et que leur expression entend désormais se libérer de tout «prêt-àfaire » et de tout «prêt-à-penser».

Cet homme libre et, par-là, fragile, se veut en outre un homme total et pas seulement un être de raison. Le corps, les émotions, la sensibilité retrouvent leur droit de cité à côté de l'esprit et de l'intelligence (c'est d'ailleurs ce dont manifestent aussi les évolutions du religieux). Comment s'étonner dès lors de voir les formes orthogonales, lisses et simples à prétention strictement fonctionnelle, perdre de leur attrait pour faire place à la complexité et à l'ambiguïté, à la couleur et à l'ornement qui, sans exclure d'emblée l'esprit et l'intelligence, en appellent davantage aux autres dimensions humaines, avec tout ce que celles-ci ont, par définition, d'irréductible à la raison.

A ces facteurs de compréhension du postmodernisme architectural et urbanistique (l'homme libre et l'homme total) s'ajouterait le fait qu'aujourd'hui, même si c'est encore de façon relativement timide, l'hégémonie de l'Occident se voit mise en question de diverses manières. La domination économique et politique de cette partie du monde et l'idée de sa suprématie culturelle sont de plus en plus souvent contestées non seulement en paroles mais aussi dans les faits. Que l'on songe à la montée en puissance économique de toute une partie de l'Asie ou à l'apparition de l'Islam dans le jeu des affirmations identitaires et dans les systèmes d'alliances politiques, nombreux sont aujourd'hui les signes qui manifestent l'ébranlement de l'Occident et les doutes qu'il commence à développer par rapport à lui-même et à son devenir. Ne peut-on dès lors voir dans les emprunts qui caractérisent le postmodernisme une certaine reconnaissance des altérités temporelles et spatiales qu'excluait jusque il y a peu l'arrogance de sa prétention rationnelle universelle?

Ces mêmes emprunts semblent par ailleurs renvoyer à une autre caractéristique bien connue de la société contemporaine: le rétrécissement du temps et de l'espace, c'est-à-dire la globalisation fonctionnant sur des systèmes de flux. Sur base de celle-ci se développe sans doute un type d'universalisme qui diffère fondamentalement de celui qui caractérisait la modernité. Ce dernier se concevait sur un mode d'homogénéité et d'abstraction. D'où des formes identiques partout à travers le 
monde et semblables pour toutes les fonctions que celles-ci abritaient. L'universalisme qui accompagne la globalisation actuelle est, affirme Lash, plus « humaniste et 'sittlich', c'est-à-dire fondé dans des pratiques sociales et dans des formes de vie concrètes » (Lash, 1990: 66-67) et par-là diversifiées. Les emprunts à des temps et à des lieux autres, l'éclectisme des styles traduirait ainsi la reconnaissance des spécificités de la vie concrète des hommes dans des temps et dans des lieux différents, sans les inscrire dans un «devoir être» tout aussi dogmatique qu'utopique.

L'évocation de la globalisation conduit encore à une autre piste d'interprétation. Si les définitions de celle-ci sont multiples et insistent plus particulièrement sur l'un ou l'autre de ses aspects - le politique chez Meyer, la communication chez Luhmann, le culturel chez Robertson, ... - aucune d'entre elles n'élude (comment le pourraient-elles?) son essentielle dimension économique. L'architecture et l'urbanisme postmodernes ne sont pas étrangers à celle-ci. En effet, l'économie contemporaine est, entre autres choses, marquée par le décloisonnement des espaces économiques, par la différenciation des produits en référence notamment aux particularités locales (l'économie des flux n'est pas indifférente aux lieux) et par l'importance accrue qu'y revêt l'extra-économique, en particulier les divers systèmes de relations sur lesquelles repose désormais fortement l'efficacité économique (Veltz, 1996). C'est aussi une économie au sein de laquelle tout ce qui est culturel tend à devenir marchandise et où le ludique et l'événementiel occupent une place privilégiée. La brève description que j'ai proposée de l'architecture et de l'urbanisme postmodernes semble pleinement en phase avec cette économie: décloisonnement des temps et des lieux, redécouverte des spécificités locales, importance des émotions et des diverses dimensions de l'humain, souci de la mise en relation des fonctions et des espaces, recherche des effets de surprise, du jeu sur les formes et sur le rapport fond/forme, des pastiches, des situations ironiques et du spectaculaire, ... Et l'on sait combien l'esthétique des villes participe aujourd'hui à leur attractivité économique et à leur insertion dans des réseaux internationaux au sein desquels elles font sens dans une logique des flux, indépendantes des lieux physiques où elles s'inscrivent.

Réalité problématique qui ouvre ses potentialités à un homme libre qui veut que soient prises en compte ses diverses dimensions et qui doit affronter dans la fragilité de cette liberté les risques inhérents aux choix qu'il est contraint de faire et que ne balisent plus guère des dispositifs institutionnels. Délitement de la position hégémonique de l'Occident et mise en équivalence sinon en supériorité de cultures autrefois subordonnées. Rétrécissement du temps et de l'espace dans un contexte globalisé où les diversités culturelles sont présentes partout à la fois. Développement d'une économie de flux qui s'empare des lieux en les transformant et dont la performance dépend de plus en plus des dimensions relationnelles, de l'existence de milieux d'interconnaissance, de l'ambivalence des réseaux et d'une innovation de rupture (Veltz, 1996: 159) produite par les rencontres imprévues, la conciliation d'inconciliables et des «courts-circuits» improbables (Veltz, 1996: 213). Telles sont, me semble-t-il, une série de caractéristiques de la société contemporaine dont on trouve l'expression dans l'architecture et l'urbanisme postmodernes.

Peut-on dès lors parler du relativisme de ceux-ci? Sans doute, diront les pessimistes. Sans conteste, insistera une certaine pensée de gauche - pas toute 
celle-ci - qui, à la suite d'Habermas, y voit une tentative réactionnaire et une menace pour les acquis d'une modernité inachevée. Peut-être plus de nuances s'imposent-elles. Tout d'abord parce qu'au-delà de leurs apparences, l'architecture et l'urbanisme postmodernes sont en connivence avec le monde où ils se déploient. En ce sens, ils ne relèveraient pas du «tout est bon» mais seraient au contraire pleinement en phase avec une nouvelle étape de la logique capitaliste qu'ils aideraient à se déployer. La critique de gauche trouverait alors sa pleine justification non parce que le post-modernisme serait relativiste mais parce qu'il exprimerait une nouvelle ruse de cette logique. Plus de nuances aussi dans la mesure où il est peut-être trop tôt encore pour statuer et ce d'autant plus que la complexité même du post-modernisme l'ouvre à divers possibles. C'est en effet l'ambivalence et l'anti-totalitarisme qui semblent peut-être en être les caractéristiques premières. A partir de là, on peut s'interroger et se demander vers où il conduit: «Est-il, comme le prétendent ses détracteurs, une entreprise de liquidation hâtive de la modernité, un laxisme soi-disant pluraliste? [...]. Ou bien un nouvel irrationalisme qui laisse la porte ouverte à toutes les réactions et à toutes les régressions? [...] (Ou encore) est-il une critique de la rationalité scientifique et technique, une déconstruction du sujet 'raisonnable' destinée à permettre la reconstruction d'une rationalité élargie, ouverte aux figures de l'Autre de la raison?» (Le Rider, 1991: 290). Dans ce dernier cas, le post-modernisme pourrait être ultramoderne parce qu'il ferait place au respect des différences, à la reconnaissance des altérités et au polythéisme des valeurs avec tout ce que Weber y voyait de contradictions mais avec aussi tout l'espoir de tolérance et de liberté qu'il peut apporter.

\section{Université catholique de Louvain}

\section{RÉFÉRENCES BIBLIOGRAPHIQUES}

Bourdin, Alain (2002), «La gare de Lille-Europe » in: Cahiers de l' Urbanisme, Ministère de la Région Wallonne/Mardaga, sept. 2002, p. 12.

Choay, Françoise (1967), «Sémiologie et urbanisme» in: Architecture d'aujourd' hui, $\mathrm{n}^{\circ} 132$.

Comte-Sponville, André (1999), Le gai désespoir, Liège, Ed. Alice.

Eisenman, Peter (1976), «Post-Functionalism» in: Oppositions 6, Fall, pp. ii-iii.

Hudnut, Joseph (1945), «The Post-Modern House » in: Architectural Record, pp. 70-75.

Jencks, Charles (1977), Mouvements modernes en architecture, Liège, Mardaga. Le Langage de l' Architecture Post-Moderne, Academy Editions-Denoël.

Lash, Scott (1990), «Post-Modernism as Humanism?» in: Bryan S. Turner ed., Theories of Modernity and Post-Modernity, London, Sage, pp. 62-74.

Le Rider, Jacques (1991), «La post-modernité » in: Commentaire, vol. 14, pp. 283-291.

Prigogine, Ilya (1998), De l'être au devenir, Liège, Ed. Alice.

Rosenberg, Harold (1965), The Tradition of the New, Mc Graw-Hill Paperback.

Simmel, Georg (1988), La tragédie de la culture, Paris, Petite Bibliothèque, Rivages.

Veltz, Pierre (1996), Mondialisation, villes et territoires. L'économie d'archipel, Paris, PUF. 